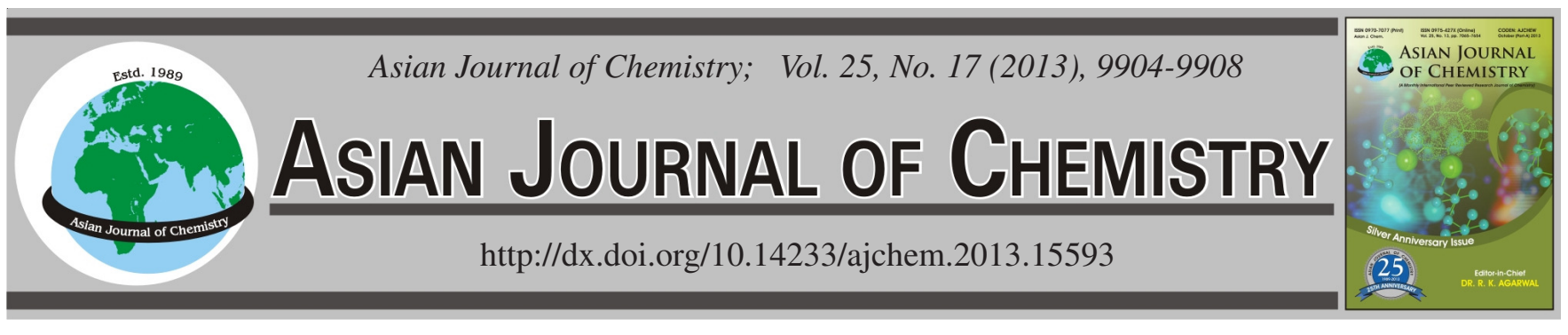

\title{
Preparation and Characterization of Hydrophobic Alginate Derivative Nanocapsules Entrapping $\lambda$-Cyhalothrin
}

\author{
Wentao Hu ${ }^{1}$, Jiacheng Li $^{1}$, Hongwei Hou ${ }^{1}$, Huigiong Yan ${ }^{1}$, Yuhong Feng ${ }^{1, *}$, Xue Mi ${ }^{1}$ and Qiang Lin ${ }^{2, *}$
}

${ }^{1}$ College of Materials and Chemical Engineering, Hainan University, Haikou 570228, P.R. China

${ }^{2}$ Hainan Normal University, Haikou 571100, Hainan, P.R. China

*Corresponding authors: Fax: +86 898 66183628; Tel: +86898 66187313; E-mail: linqiang_1962@163.com; ljcfyh@gmail.com

\begin{abstract}
Hydrophobic alginate derivative was synthesized by the amidation of alginate and octylamine. The generated octyl-grafted alginateamide was confirmed by FTIR and ${ }^{1} \mathrm{H}$ NMR. The degree of substitution was 0.23 and whose critical aggregation concentration in aqueous medium was $0.34 \mathrm{mg} / \mathrm{mL}$. This strategy of nanocapsule formulation was based on microemulsion template with octyl-grafted alginateamide derivative. The morphology and the size of nanocapsules were characterized by transmission electron microscopy, whose average size is $25.78 \mathrm{~nm}$. The nanocapsule has a high loading efficiency valve of $99.95 \%$. The release characteristics of the nanocapsules were evaluated in methanol and the results indicated that the nanocapsules restrained the release of $\lambda$-cyhalothrin.
\end{abstract}

Key Words: Alginate, Octyl-grafted alginate-amide derivative, Polyoxyethylene styrenyl phenyl ether, $\lambda$-cyhalothrin, Nanocapsule. |

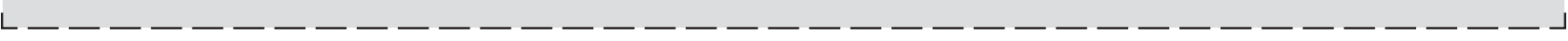

\section{INTRODUCTION}

Alginate is obtained from algal or bacterias, which is a polyanionic linear polysaccharide consisting of $1,4-\beta-\mathrm{D}-$ mannuronic acid (M block) and C-5 epimer $\alpha$-L-guluronic acid ( $\mathrm{G}$ block) units through different proportions of arrangements in GG, MG and MM blocks ${ }^{1}$. Alginate has some special properties, such as biocompatibility, non-toxicity and biodegradability $^{2}$, in addition, the ability to form hydrogel by the interactions between $\mathrm{G}$ blocks of alginate and divalent cation ${ }^{3}$. Thus, alginate has been widely used to make hydrogel and microsphere for drug delivery ${ }^{4-8}$. However, its utilization in the formation of oil-in-water $(\mathrm{o} / \mathrm{w})$ microemulsion (microcapsule) is very less due to its water-solubility ${ }^{9}$. It has been reported that alginate derivative is a good candidate for the formation of nanoparticle because of its amphiphilic by introducing the hydrophobic groups into the hydrophilic polysaccharide $^{10,11}$. For instance, the hydrophobic segments of alginate can undergo an intermolecular interaction to form clusters through the covalent modification of alginate by hydrophobic materials ${ }^{12}$ which is an effective way to enhance the stability, loading capacity and efficiency of microemulsion. In addition, the utilization of alginate is greatly increased because numerous hydrophobical modified alginate derivatives gathering at the surfaces of the microemulsion droplets ${ }^{13}$.

$\lambda$-Cyhalothrin is a kind of pyrethroid insecticide and has been widely used in controlling insect pests in agriculture ${ }^{14}$.
Among the numerous formulations of pesticide, oil-in-water $(\mathrm{o} / \mathrm{w})$ microemulsion has some advantages of reducing organic solvent used and improving the safety and stability during storage and transportation ${ }^{15}$. Nevertheless, the microemulsion is unstable which may lead to the membrane damage and the rapidly release of core substances to the farmland ${ }^{16}$ resulting in large number of pesticide residues, which are considered dangerous to the environment and not environmental benign. Therefore, it is crucial to avoid or minimize the deficiencies mentioned above. One possible way is to prepare a nanocapsule of core-shell structure $^{17}$. We encapsuled the hydrophobic pyrethroid by the appropriate polymers to improve the stability ${ }^{18}$ and decrease the possibility of direct contact with people as well as the burst ${ }^{19}$.

In this paper, a nanocapsule from polyoxyethylene styrenyl phenyl ether and amphiphilic alginate derivative as a carrier for the application of $\lambda$-cyhalothrin is prepared and charactrized. An amphiphilic alginate-amide derivative was prepared by octylamine grafting onto the carboxylic group of alginate. The formulation of nanocapsule containing $\lambda$-cyhalothrin was based on microemulsion template. Besides, the effection on the release behaviours of pesticides by the addition of surfactant and octyl-grafted amphiphilic alginateamide derivative has been investigated.

\section{EXPERIMENTAL}

Sodium alginate, octyl amine, 1-ethyl-3-(3-dimethylaminopropyl) carbodiimide hydrochloride (EDC $\cdot \mathrm{HCl}), \mathrm{HCl}$, 
ethanol, methanol and pyrene were commercial available and purchased from Aladdin Chemical Reagent (Shanghai, China). The chemicals were analytical grade and used without further purification. Polyoxyethylene styrenyl phenyl ether and $\lambda$-cyhalothrin $\left(\mathrm{C}_{23} \mathrm{H}_{19} \mathrm{ClF}_{3} \mathrm{NO}_{3},>94.6 \%\right)$ were provided by Yangnong Chemical (Jiangsu, China).

Preparation of octyl-grafted alginate-amide derivative: Octyl-grafted alginate-amide derivative (OAD) was prepared by the amidation through alginate coupling with octylamine in the presence of $\mathrm{EDC} \cdot \mathrm{HCl}^{13,20}$. First of all, NaAlg $(3.0 \mathrm{~g})$ was dissolved in a volume of $90 \mathrm{~mL}$ of water. Then, $\mathrm{EDC} \cdot \mathrm{HCl}$ $(0.73 \mathrm{~g})$ was added to the solution. The $\mathrm{pH}$ value of the solution was set at 3.4 by hydrochloric acid $(0.5 \mathrm{~mol} / \mathrm{L})$ and the alginate solution was diluted to a concentration of $2.5 \mathrm{wt} \%$ with distilled water. The amidation reaction was carried out by adding octyl amine $(5.1 \mathrm{~mL})$ and stirred at $35^{\circ} \mathrm{C}$. After $24 \mathrm{~h}$, $300 \mathrm{~mL}$ of ethanol $(95 \% \mathrm{v} / \mathrm{v})$ was added to precipitate the product. Then the mixture was centrifuged, washed with $100 \mathrm{~mL}$ ethanol $(95 \% \mathrm{v} / \mathrm{v})$ and dried at $60{ }^{\circ} \mathrm{C}$ to obtain the product. The product was dialyzed in distilled water for 3 days. At last, octyl-grafted alginate-amide derivative was precipitated from the solution by adding $300 \mathrm{~mL}$ ethanol (95\% v/v) and then centrifuged and dried once again.

The degree of substitution (DS) of OAD was calculated based on the N/C ratio and determined by an vario EL cube elemental analyser (Elementar) and all samples were analyzed in duplicate. It was measured by the following eqn. 1 .

$$
\mathrm{D}=\frac{6 \alpha \times \mathrm{M}_{\mathrm{C}}}{\mathrm{M}_{\mathrm{N}}-8 \alpha \times \mathrm{M}_{\mathrm{C}}}
$$

$M_{N}$ is the the molecular weight of nitrogen. $M_{C}$ is the the molecular weight of carbon. D is the degree of substitution (DS) of OAD. $\alpha$ is the the N/C ratio of OAD.

Fourier transform infrared and ${ }^{1} \mathrm{H}$ nuclear magnetic resonance spectroscopy: The presence of an amido bond and octyl groups of the octyl-grafted alginate-amide derivative was confirmed by the fourier transform infrared (FT-IR) and ${ }^{1} \mathrm{H}$ nuclear magnetic resonance $\left({ }^{1} \mathrm{H}\right.$ NMR). FTIR spectra of alginate and OAD were recorded on a Tensor27 Fourier transform infrared spectrometer (Bruker). The samples were mixed with $\mathrm{KBr}$ and compressed to semitransparent disks for spectroscopic analysis.

${ }^{1} \mathrm{H}$ NMR was performed on a DMX500 nuclear magnetic resonance spectrometer (Bruker). The samples were dissolved in $\mathrm{D}_{2} \mathrm{O}(99.9 \%)$ to the concentration of $c a .10 \mathrm{mg} / \mathrm{mL}$.

Fluorescence measurement: The critical aggregation concentration (CAC) of OAD was determined by fluorescence measurement $\mathrm{t}^{21}$. Pyrene, as a hydrophobic probe, was dissolved in ethanol to a final concentration of $0.04 \mathrm{mg} / \mathrm{mL}$. $40 \mu \mathrm{L}$ of diluted pyrene was then added into the tube and the ethanol was volatilized by nitrogen gas. $10 \mu \mathrm{L}$ of OAD solution with varied concentrations from $1 \times 10^{-4}$ to $10 \mathrm{mg} / \mathrm{mL}$ were added into the tube. The mixtures were sonicated for $40 \mathrm{~min}$ and shook in a shaking water bath overnight at $30^{\circ} \mathrm{C}$. Pyrene emission spectrum was obtained using a F7000 fluorescence spectrophotometer (Hitachi). The probe was excited at 335 $\mathrm{nm}$ and the emission spectrum was collected in the range of $335-600 \mathrm{~nm}$ at an integration time of $1 \mathrm{~s}$. The excitation and emission slit openings were $2.5 \mathrm{~nm}$.
Preparation of nanocapsule: The preparation of $\lambda$ cyhalothrin-loaded hydrophobic alginate derivative nanocapsules was based on microemulsion. Polyoxyethylene styrenyl phenyl ether (602\#) is a nonionic emulsifier and plays a key role in the formation of microemulsions ${ }^{21} .2 .5 \mathrm{~g} \lambda$-cyhalothrin and $18 \mathrm{~mL} \mathrm{602 \#} \mathrm{were} \mathrm{introduced} \mathrm{into} 60 \mathrm{~mL}$ of deionized water and stirred at $60{ }^{\circ} \mathrm{C}$ for $1 \mathrm{~h}$. Then $20 \mathrm{~mL}$ OAD solution (5.0 $\mathrm{mg} / \mathrm{mL}$ ) was added to the aqueous solution and stirred for $0.5 \mathrm{~h}$. Finally, the nanocapsules solution was prepared by slowly dripping $20 \mathrm{~mL} \mathrm{CaCl}_{2}$ solution $(2.5 \mathrm{mg} / \mathrm{mL})$ to the oil-in-water microemulsion system with stirring for $0.5 \mathrm{~h}$.

Transmission electron microscopy and particle size distribution: The morphology of nanocapsules was examined with a JEM 2100 transmission electron microscopy (TEM) at an acceleration voltage of $100 \mathrm{kV}$. The nanocapsules were placed on a copper grid and the excess fluid was air-dried. The samples were negatively dyed in a $2 \%$ phosphotungstic acid solution and then dried with an infrared lamp before observation. The size distribution of the nanocapsules was statistically analyzed by the graph of TEM.

Determination of loading efficiency: The amount of unencapsulated $\lambda$-cyhalothrin was determined through a crystallization method. $5 \mathrm{~mL}$ of the solutions of nanocapsule and microemulsion were added to a $10 \mathrm{~mL}$ gas-tight vial, respectively, cooled at $4{ }^{\circ} \mathrm{C}$ for $2 \mathrm{~h}$ and centrifuged to remove the precipitated unencapsulated $\lambda$-cyhalothrin from the solution. The precipitated $\lambda$-cyhalothrin was then dissolved by $5 \mathrm{~mL}$ of hexane, stirred for $10 \mathrm{~min}$. The $\lambda$-cyhalothrin content in the hexane was measured by GC analysis with a $6890 \mathrm{~N}$ gas chromatography instrument (Agilent). The $\lambda$-cyhalothrin loading efficiency was equal to the ratio of loaded amount to the total amount of $\lambda$-cyhalothrin.

Release studies: The release experiment of $\lambda$-cyhalothrin from nanocapsules was performed similarly to the method described by Kumbar et al. ${ }^{23}$. Methanol was used as dissolvent to evaluate the release of $\lambda$-cyhalothrin. $20 \mathrm{~mL}$ of $\lambda$-cyhalothrinloaded nanocapsules were introduced into a dialysis bag (cutoff 7,000MW). The bag was immersed into $230 \mathrm{~mL}$ methanol at $40^{\circ} \mathrm{C}$ with agitation $(100 \mathrm{rpm})$. At predetermined intervals, samples of $0.1 \mathrm{~mL}$ were taken and analyzed by GC analysis.

\section{RESULTS AND DISCUSSION}

Synthesis and characterization of octyl-grafted alginateamide derivative: Octyl-grafted alginate-amide derivative was successfully prepared by the introduction of octylamine groups into alginate. New property was obtained and the special properties of alginate was kept as well ${ }^{24}$. Data collected by elemental analysis showed the substitution was 0.23 . The free primary amine groups of octylamine reacted with the carboxylic acid groups on the alginate backbones induced by $\mathrm{EDC} \cdot \mathrm{HCl}$ (Fig. 1).

The presence of an amido linkage and octyl group was confirmed by FTIR (Fig. 2). The peak at $1031 \mathrm{~cm}^{-1}$ was assigned to the stretching vibration of C-O-C, the peak at $1414 \mathrm{~cm}^{-1}$ was attributed to the symmetric stretching vibration of carboxyl group and the he peak at $1631 \mathrm{~cm}^{-1}$ was assigned to asymmetric stretching vibration of carboxyl group. It should be further noted that the peak shifts from $1613 \mathrm{~cm}^{-1}$ (B) to $1631 \mathrm{~cm}^{-1}$ (A) 


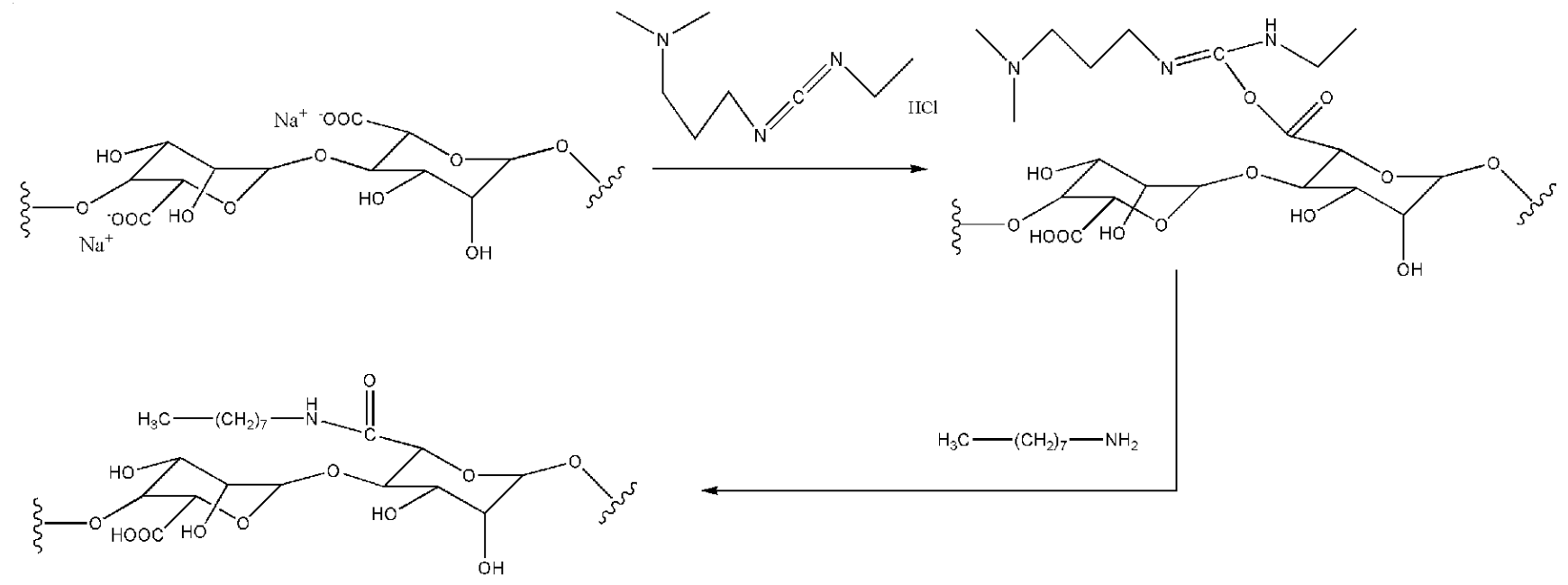

Fig. 1. Synthesis of octyl-grafted alginate-amide derivative

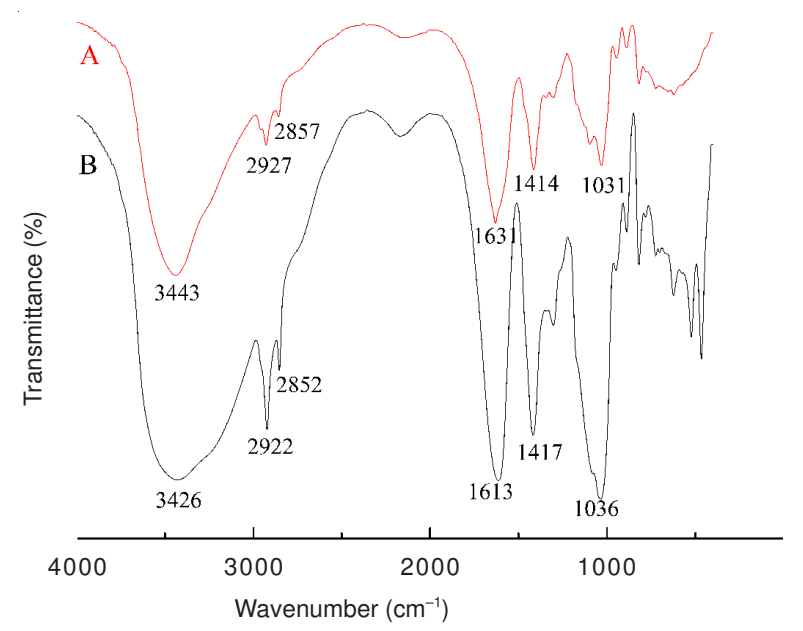

Fig. 2. FT-IR spectra of OAD (A) and alginate (B)

indicated the presence of octyl groups. The peaks at 2927 and $2857 \mathrm{~cm}^{-1}$ were assigned to the $\mathrm{C}-\mathrm{H}$ stretching vibration of methyne groups. The broad peak at $3426 \mathrm{~cm}^{-1}$ increased to sharp band at $3443 \mathrm{~cm}^{-1}$ representing the O-H stretching vibrations of hydroxyl group and the $\mathrm{N}-\mathrm{H}$ stretching vibrations of amide group overlapped with each other.

The successful graft of alginate octyl groups was further demonstrated by ${ }^{1} \mathrm{H}$ NMR (Fig. 3). Compared with the spectrum of $\mathrm{NaAlg}$, the proton peaks within the region of 3.8-5.2 ppm indicated the presence of native alginate carbons ${ }^{13}$. The emerged new and increased peaks observed from 0.9-3.6 ppm proved the presence of new groups linking to alginate. The methyl and methylene protons assignment of the octyl graft is as follows (Fig. 3A): $\delta 3.0=\mathrm{CH}_{2}$ (carbon 1 of octyl graft); $\delta 1.4=\mathrm{CH}_{2}$ (carbons 2-7 of octyl graft); $\delta 0.9=\mathrm{CH}_{3}$ (carbon 8 of octyl graft).

Formation of nanoparticles: The ratio of I372/I383 from fluorescence spectra of pyrene at varied concentrations of OAD (A) and alginate (B) as shown in Fig. 4. With increasing concentration of native alginate, basically no obvious decline in the I372/I383 valves was found when the OAD concentration was less than $0.1 \mathrm{mg} / \mathrm{mL}$. Nevertheless, when the OAD concentration increased to $0.3 \mathrm{mg} / \mathrm{mL}$, the ratio of I372/I383 declined obviously and when the concentration of OAD reached
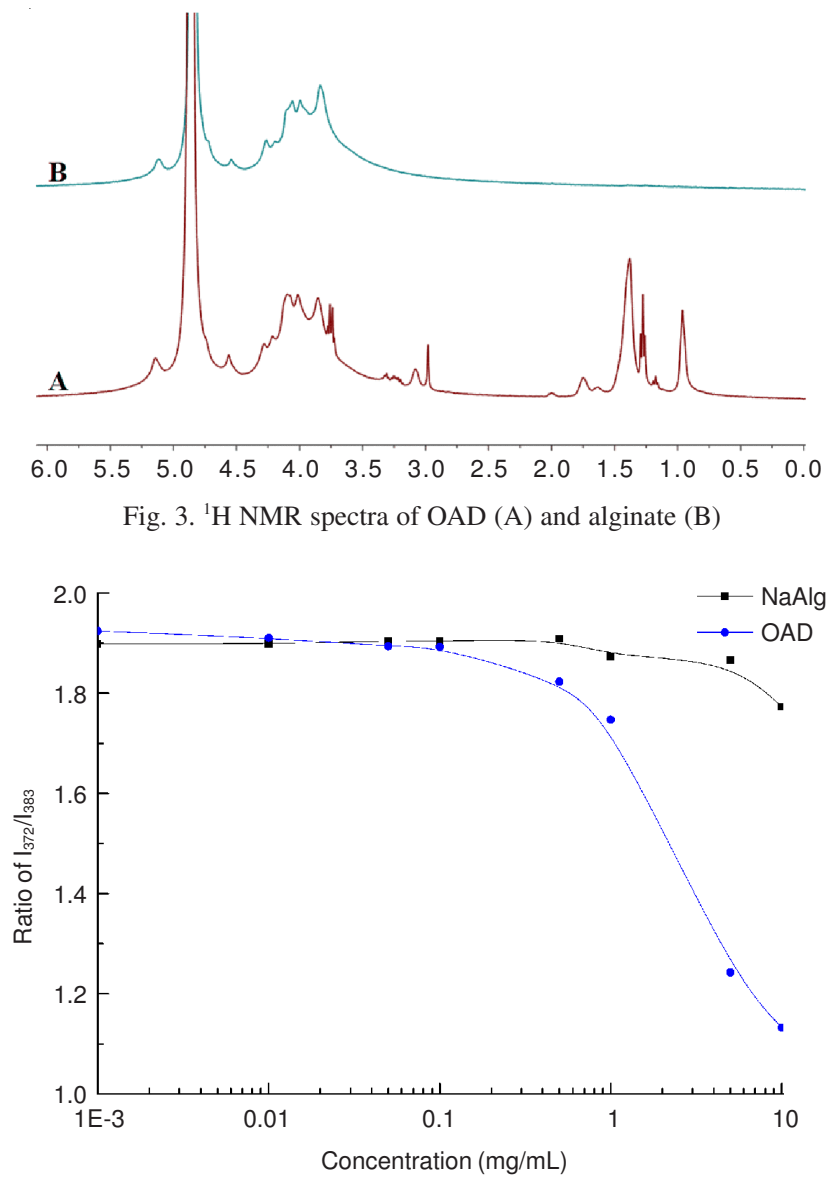

Fig. 4. Profile of ratios of I372/I383 versus varied concentrations of OAD and $\mathrm{NaAlg}$

to $10 \mathrm{mg} / \mathrm{mL}$, a sharp decline in the ratio was observed, indicating that the pyrene is transferred from aqueous media to the hydrophobic microdomains.

The critical aggregation concentration $(\mathrm{CAC})$ is the threshold concentration of self-aggregation formation ${ }^{21}$. The value of the OAD was $0.337 \mathrm{mg} / \mathrm{mL}$ determined by the interception of two straight lines (the low concentration ranges and the high concentration ranges). Hence, when the concentration of OAD exceeded $0.337 \mathrm{mg} / \mathrm{mL}$, the nanoparticle can be formed through the hydrophobic interactions between octyl groups. 
This strategy of nanocapsule formulation was based on microemulsion template. Firstly, It is an optically clear solution since the $\lambda$-cyhalothrin molecule diffused and entered into the hydrophobic microdomain of self-aggregates process of 602\#. Secondly, the hydrophobic chains of OAD were inserted into the microemulsion through the intermolecular hydrophobic interaction between octyl group and $\lambda$-cyhalothrin ${ }^{25}$. Thirdly, the interactions between G-blocks of alginate and divalent cation $^{3}$ occurred. The shell was formed by the association of OAD with $\mathrm{Ca}^{2+}$ (Fig. 5).

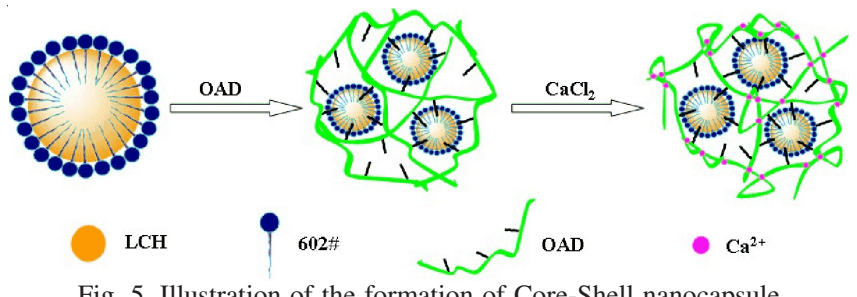

Fig. 5. Illustration of the formation of Core-Shell nanocapsule

Morphology and size of nanocapsules: The size distribution of the prepared nanoparticles was analyzed by performing TEM (Fig. 6A). It is shown that the average diameter of nanoparticle was $7.40 \mathrm{~nm}$. It was also indicated that the nanoparticles had spherical structures, which displayed a unimodal particle size distribution.
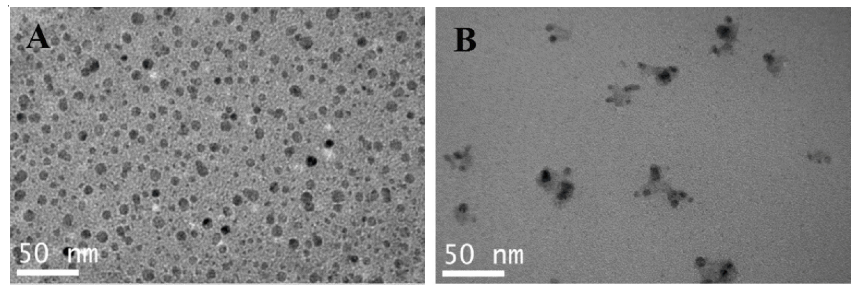

Fig. 6. TEM of nanoparticle prepared with $2.5 \% \lambda$-cyhalothrin $+18 \%$ $602 \#+79.5 \% \mathrm{H}_{2} \mathrm{O}(\mathrm{A}) ; 2.5 \% \lambda$-cyhalothrin $+18 \% 602 \#+1 \mathrm{mg} /$ $\mathrm{mLOAD}+0.5 \mathrm{mg} / \mathrm{mLCaCl}_{2}+79.5 \% \mathrm{H}_{2} \mathrm{O}(\mathrm{B})$

The nanocapsules were confirmed by TEM (Fig. 6B), in this figure, the black spots represent the $\lambda$-cyhalothrin nanoparticles appearing inside of the gray area (602\# and OAD membrane). The irregular morphology of nanocapsules probably due to water evaporation in the membranes when drying the sample. The average size of the nanocapsules was $25.78 \mathrm{~nm}$.

$\boldsymbol{\lambda}$-Cyhalothrin loading efficiency: The transparent nanocapsules solution of $\lambda$-cyhalothrin $(2.5 \%)$ was successfully prepared. The loading efficiency of nanocapsule and microemulsion were 99.5 and $99.8 \%$, respectively. Microemulsion is suitable for trapping hydrophobic substance. The $\lambda$-cyhalothrin molecules were solubilized in the solution of $602 \#$ to achieve a homogeneous solution. The formation of nanocapsule based on microemulsion template was an effective method, which inherited a high loading efficiency.

$\boldsymbol{\lambda}$-Cyhalothrin release evaluation: Release profile of $\lambda$-cyhalothrin was investigated by comparing the release of nanocapsule (B) with that of microemulsion (A) (Fig. 7), the release of $\lambda$-cyhalothrin decreased with the existence of OAD. The results could be explained by the fact that the shell was formed by the introduction of $\mathrm{OAD}$ and $\mathrm{CaCl}_{2}$ and the diffusion of $\lambda$-cyhalothrin molecules from the nanocapsules was restrained.

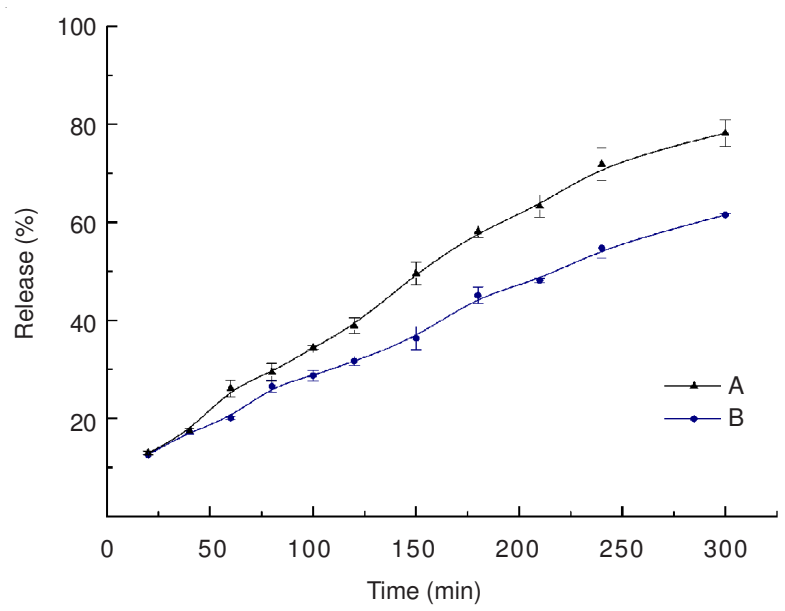

Fig. 7. Profile of release of $\lambda$-cyhalothrin from the nanoparticles prepared with $2.5 \% \lambda$-cyhalothrin $+18 \% 602 \#+79.5 \% \mathrm{H}_{2} \mathrm{O}(\mathrm{A}) ; 2.5 \% \lambda$ cyhalothrin $+18 \% 602 \#+1 \mathrm{mg} / \mathrm{mLOAD}+0.5 \mathrm{mg} / \mathrm{mLCaCl}_{2}+$ $79.5 \% \mathrm{H}_{2} \mathrm{O}(\mathrm{B})$

To simplify the analysis the effect of OAD and $\mathrm{Ca}^{2+}$ on the mechanism of $\lambda$-cyhalothrin release from the nanocapsules, the release data was analyzed by applying the empirical equation ${ }^{26}$ :

$$
\mathrm{kt}^{\mathrm{n}}=\frac{\mathrm{M}_{\mathrm{t}}}{\mathrm{M}_{\infty}}
$$

$\mathrm{M}_{t} / \mathrm{M}_{\infty}$ is the fractional release of drug in time $\mathrm{t}$, provided that $\mathrm{t}$ is limited to times where $\mathrm{M}_{\mathrm{t}} / \mathrm{M}_{\infty}<0.6$. $\mathrm{k}$ is the constant characteristic of the delivery system and $n$ is the diffusion exponent characteristic of the release mechanism. For normal Fickian diffusion the value of $n=0.5$. Case II diffusion the value of $n$ $=1.0$. The value of $\mathrm{n}$ intermediate between the above limits indicate Non-Fickian or anomalous transport.

By applying least-squares to the release data, the estimated correlation coefficient (r) values are presented in Table-1. According to these data, the values of $\mathrm{n}$ were 0.605 and 0.707 , indicating that the mechanism of release from anomalous transport.

TABLE-1

RESULTS OF $\mathrm{k}, \mathrm{n}$ AND R CALCULATED WITH eqn. 3

\begin{tabular}{ccccc}
\hline Sample & $\mathrm{k}$ & $\mathrm{n}$ & $\mathrm{R}$ & $\begin{array}{c}\text { Diffusion } \\
\text { mechanism }\end{array}$ \\
\hline $\begin{array}{c}\text { Nanoemulsion } \\
\text { (A) }\end{array}$ & 1.406 & 0.707 & 0.992 & $\begin{array}{c}\text { Anomalous } \\
\text { transport }\end{array}$ \\
$\begin{array}{c}\text { Nanocapsule } \\
\text { (B) }\end{array}$ & 1.86 & 0.605 & 0.994 & $\begin{array}{c}\text { Anomalous } \\
\text { transport }\end{array}$ \\
\hline
\end{tabular}

\section{Conclusion}

Amphiphilic alginate derivative was successfully prepared by the amidation of alginate with octylamine. The emulsification-gelation technique for the preparation of $\lambda$-cyhalothrin nanocapsules is proved to be effective and practical. $\lambda$ Cyhalothrin as a hydrophobic core was successfully encapsulated with 602\# and OAD and the release rate collected indicated a prolonged behaviour. The nanocapsule can be used as a potential carrier for restraining the release of hydrophobic pesticide. 


\section{ACKNOWLEDGEMENTS}

Financially supported from the Project "The Research and Industrialization of Green Ecological Pesticide" (2011BAE06A06-07, 2011BAEO6B04-7), Plan of Hainan province supported by major scientific and technological projects (ZDXM20120003) and the support from Analytical and Testing Center of Hainan University was gratefully acknowledged.

\section{REFERENCES}

1. H. Bu, G.T.M. Nguyen and A.L. Kjøniksen, Polym. Bull., 57, 563 (2006).

2. A. Smelcerovic, Z. Knezevic-Jugovic and Z. Petronijevic, Curr. Pharm. Design, 14, 3168 (2008)

3. L. Li, Y. Fang, R. Vreeker and I. Appelqvist, Biomacromolecules, 8, 464 (2007).

4. H. Zhu, R. Srivastava and M.J. McShane, Biomacromolecules, 6, 2221 (2005).

5. V.R. Babu, M. Sairam and K.M. Hosamani, Carbohydr. Polym., 69 241 (2007).

6. M. George and T.E. Abraha, J. Control. Rel., 114, 1 (2006).

7. A.K. Anal and W.F. Stevens, Int. J. Pharm., 290, 45 (2005).

8. J.Y. Wang, Y. Jin, R. Xie, J.Y. Liu, X.J. Ju, T. Meng and L.Y. Chu, J. Colloid Interf. Sci., 353, 61 (2011).

9. M. Leonard, M.R.D. Boisseson, P. Hubert, F. Dalencon and E. Dellacheriea, J. Control. Rel., 98, 395 (2004).
10. L. Yang, B. Zhang, L. Wen, Q. Liang and L.M. Zhang, Carbohydr. Polym., 28, 218 (2007).

11. A.G. Cunha and A. Gandini, Cellulose, 17, 1045 (2010).

12. Q. Li, C.G. Liu, Z.H. Huang and F.F. Xue, J. Agric. Food Chem., 59, 1962 (2011).

13. J.S. Yang, H.B. Ren and Y.J. Xie, Biomacromolecules, 12, 2982 (2011).

14. J. Liu, X.M. Lv, J.M. Xie, Y.F. Chu, C. Sun and Q. Wang, Environ. Sci. Pollut. Res., 16, 414 (2009).

15. C.P. Chin, C.W. Lan and H.S. Wu, Ind. Eng. Chem. Res., 51, 4710 (2012).

16. W.T. Kim, H. Chung, I.S. Shin, K.L. Yam and D.H. Chung, Carbohydr. Polym., 71, 566 (2008)

17. Q. Zhang, P.P. Zhang and Q.Z. Jiao, Chem. Res. Chin. Univ., 22, 379 (2006).

18. G.C. Mak, K.Y. Cheung and D. Trau, Chem. Mater., 20, 5475 (2008).

19. N. Anton, J.P. Benoit and P. Saulnier, J. Control. Rel., 128, 185 (2008).

20. F. Vallee, C. Muller, A. Durand, S. Schimchowitsch, E. Dellacherie, C. Kelche, J.C. Cassel and M. Leonard, Carbohydr. Res., 344, 223 (2009).

21. C.G. Liu, K.G.H. Desai, X.G. Chen and H.J. Park, J. Agric. Food Chem., 53, 437 (2005).

22. M. Okubo, Y. Furukawa, K. Shiba and T. Matoba, Colloid Polym. Sci., 281, 182 (2003).

23. S.G. Kumbar, A.M. Dave and T.M. Aminabhavi, J. Appl. Polym. Sci., 90, 451 (2003)

24. S.N. Pawar and K.J. Edgar, Biomaterials, 33, 3279 (2012).

25. D. Maysinger, Org. Biomol. Chem., 5, 2335 (2007).

26. P.L. Ritger and N.A. Peppas, J. Control. Rel., 5, 37 (1987). 